\title{
Exploring the potential effect and mechanisms of protocatechuic acid on human hair follicle melanocytes
}

BO LI

JUN TAN

BOSHENG ZOU

XIAOJIA LIU

YILING YU*

Department of plastic and aesthetic laser surgery, The people's Hospital of Hunan province, 1st Affiliated Hospital of Hunan Normal University Changsha 410005, Hunan, China

Accepted January 24, 2020

Published online February 20, 2020

\begin{abstract}
This study aims to evaluate the effect of protocatechuic acid (PCA) on human hair follicle melanocytes (HFM). Normal primary HFM were isolated and cultured till logarithmic period of second passage, then treated with different concentrations of PCA $\left(0.1-200 \mu \mathrm{mol} \mathrm{L}^{-1}\right)$ to study the cell proliferation, melanin contents, tyrosinase activity and protein and mRNA expression of melanogenic genes (tyrosinase-related protein 1 (TRP-1), tyrosinase-related protein 2 (TRP-2), and microphthalmia-associated transcription factor (MITF)) in the cultured HFM. In addition, we have also measured the contents of superoxide dismutase (SOD) and glutathione (GSH) in PCA treated HFM. Vitamin C was used as a positive control. The result showed that PCA can decrease the synthesis of melanin and the tyrosinase activity with $I C_{50}=8.9 \mu \mathrm{mol} \mathrm{L}^{-1}$ and $I C_{50}=$ $6.4 \mu \mathrm{mol} \mathrm{L}{ }^{-1}$, respectively, at the treatment time of 24 hours, without inducing any cytotoxicity in HFM cells. In addition, the mRNA transcription and protein expression levels of TRP-1, TRP-2 and MITF significantly decreased with a dose-dependent manner after 24-hour PCA treated in HFM cells. Furthermore, PCA has significantly increased the SOD and GSH activity in a dose-dependent manner for 24-hour PCA treatment. This study suggested that PCA has an inhibitory effect on the production of melanin through down-regulation of the expression of melanogenesis-related protein and the effect of anti-oxidation, which could be useful for the therapy of melanin overproduction or skin whitening.
\end{abstract}

Keywords: protocatechuic acid, human hair follicle melanocytes, melanin synthesis, tyrosinase activity, anti-oxidation

Protocatechuic acid (3,4-dihydroxybenzoic acid, PCA, Fig. 1), the main metabolite of anthocyanins, is one of the effective components of many Chinese traditional medicines, widely distributed in fruits and vegetables such as tea, grape, raspberry, chicory, cauliflower, and buckwheat, but in low concentrations (1). Recent studies have reported that PCA has many pharmacological functions, such as antioxidant, anti-inflammation, anti-

\footnotetext{
*Correspondence, e-mail: yilingyu01@163.com
} 


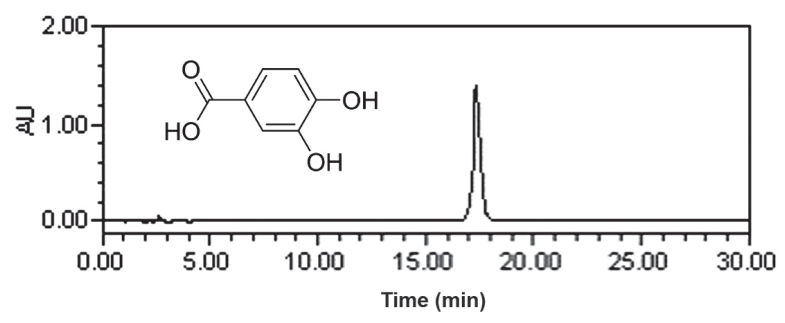

Fig. 1. The chemical structure and HPLC analysis of PCA.

diabetes, anti-infection, anti-aging, neuro-protection, anti-virus, promoting stem cell proliferation and differentiation (2, 3). In addition, PCA can scavenge 1,1-diphenyl-2-trinitrophenyl hydrazine (DPPH), nitric oxide (NO), hydrogen peroxide free radicals (4). So far, PCA is considered to be nontoxic and a relatively safe compound for oral administration.

Melanin, a pigment, is synthesized by melanocytes in the epidermis basal layer. After being synthesized, melanin is transferred to epidermal keratinocytes via dendritic processes (5). Melanin overexpression leads to hyperpigmentation, which will further cause pigmentary disorders such as melasma, freckles, and age spots (6). To address these issues, researchers have developed anti-melanogenic agents such as arbutin, kojic acid, and hydroquinone; however, many anti-melanogenic agents usually exert slight whitening effects or more severe side effects.

Anti-melanogenic agents are usually reported to target the activation or expression of tyrosinase. Tyrosinase is a complex multi-subunit copper-containing oxidoreductase, which is the key enzyme and rate-limiting enzyme for melanin synthesis in organisms. Tyrosinase inhibitors have great potential in the treatment of melanin metabolic diseases and cosmetics (7). Currently, tyrosinase inhibitors are mainly obtained from chemical synthesis or extraction of natural products. It is of great significance in practical application to find the high-efficiency and safe tyrosinase inhibitors.

As a phenolic acid, PCA has a similar parent nucleus structure to many hydroxybenzoate tyrosinase inhibitors, which suggests that PCA may be a potential tyrosinase inhibitor. Thus, in this study, we aimed to evaluate the effect of PCA on human hair follicle melanocytes (HFM) including cell proliferation, melanin synthesis, tyrosinase activity and the expression of melanogenesis-related genes, in order to find a potential therapy for melanin overexpression disease or skin whitening keeping reagent.

\section{EXPERIMENTAL}

\section{Chemicals}

EMEM culture medium (Gibco), Keratinocyte serum-free medium (KSFM, Gibco), Fetal bovine serum (FBS, Hyclone), $0.25-0.50 \%$ separation enzyme (Sigma), cholera toxin (CT, Sigma), 12-o-tetradecanoylphobol-13-acetate (TPA, Sigma), geneticin (G418, Sigma), 3-isobutyl-1-methylxanthine (IBMX, Sigma), bFGF (Sigma), methylthiazolyldiphenyl tetrazolium bromide (MTT, Shanghai Huamei Bioengineering Company), MGM (EMEM medium, 10 \% FBS, $0.2 \mu \mathrm{g} \mathrm{mL} \mathrm{g}^{-1} \mathrm{CT}, 50 \mathrm{nmol} \mathrm{L}{ }^{-1} \mathrm{TPA}$ and 50 \% KSFM), $0.1 \%$ L-dopa (Sigma). Superoxide 
dismutase (SOD) and glutathione (GSH) kits were obtained from Nanjing Jiancheng Bioengineering Institute (Nanjing, China). Anti-MITF antibody (ab20663) and anti-TRP1 antibody (ab3312) were purchased from Abcam. Anti-TRP2 antibody (sc-74439) and anti- $\beta$-actin antibody (sc-47778) were purchased from Santa Cruz Biotechnology. Protocatechuic acid was purchased from MedChemExpress LLC, USA (HY-N0294, purity $99.95 \%$ ).

\section{HPLC analysis for the purity of PCA}

The chemical structure of PCA was identified by ${ }^{1} \mathrm{H}$ and ${ }^{13} \mathrm{C}$ NMR spectral data. The purity of PCA was analyzed by HPLC-PDA method. In short, a Waters 2695 Alliance HPLC system combined with Waters 2998 PDA detector and the Scienhome Kromasil C18 column $(4.6 \mathrm{~mm} \times 250 \mathrm{~mm}, 5 \mu \mathrm{m})$ at a column temperature of $30{ }^{\circ} \mathrm{C}$ were used. The mobile phase used was (A) $0.1 \%$ formic acid in water and (B) acetonitrile, which started with 10 to $45 \%$ B in 0-10 min; 45 to $95 \%$ B in 10-30 min; 95 to $95 \%$ B in 30-35 min; 95 to $10 \%$ B in 35-40 min.

\section{Cell isolation and cell culture}

Normal human scalp (about 10-15 $\mathrm{cm}^{2}$ ) was obtained from six women (mean age 40 years) in routine plastic surgery at the people's Hospital of Hunan province (Changsha, China). The HFM isolation was done according to the method Tobin et al. had reported (8). Removal of the epidermis and upper part of dermis $1 \mathrm{~mm}$, cut into strips about $0.5 \mathrm{~cm}$ wide and 3-4 cm long; immersed in $0.25-0.50 \%$ separation enzyme solution, and stored at $4{ }^{\circ} \mathrm{C}$ for $12-16 \mathrm{~h}$. Free hair follicles were digested at $37^{\circ} \mathrm{C}$ with $0.25 \%$ trypsin for $10-15$ minutes to form a single cell suspension. Melanocyte growth medium (MGM) was added to the cells and then dripped into a $35 \mathrm{~mm}$ plastic culture dish or flask. The inoculation density was $3-5 \times 10^{4} \mathrm{~cm}^{-2}$. Then cultured for $4-5$ weeks at $37^{\circ} \mathrm{C}, 5 \% \mathrm{CO}_{2}$ incubator, and the pure HFM (about 5-10 $\times 10^{6}$ ) was obtained after 1-2 times passages. Use the differential pancreatin to remove the contaminated keratinocytes, and use G418 to remove the contaminated fibroblasts. After 1 week of culture, the morphology of HFM was observed at a high magnification microscope, and dopamine staining was performed. The study was approved by the medical ethics committee of the people's Hospital of Hunan province/1st Affiliated Hospital of Hunan Normal University, Changsha, Hunan, China. The approval form number is 15 of the 2017 scientific research ethics review.

\section{Dopamine staining}

The cells were cultured and seeded on the cover slide. Phosphate buffer saline (PBS) was used to wash the cells, followed by fixing the cells in $2 \%$ polyformaldehyde (PFA) solution for $10 \mathrm{~min}$, and then wash with PBS 3 times. $0.1 \%$ dopamine (in $0.1 \mathrm{~mol} \mathrm{~L}^{-1} \mathrm{PBS}$ solution) was used for staining at $37^{\circ} \mathrm{C}$ for $5 \mathrm{~h}$. The cell nuclei were dyed with eosin, then imaged under the bright field microscope.

\section{Effects of PCA on HFM cell proliferation}

The effect of PCA on HFM cell proliferation was measured by the methylthiazolyldiphenyl-tetrazolium bromide (MTT) assay. HFM cells were seeded at a concentration of 5000 cells per well, $200 \mu \mathrm{L}$ medium per well for 96-well plates. Then the cells were cultured 
at $37^{\circ} \mathrm{C}, 5 \% \mathrm{CO}_{2}$ incubator for $12 \mathrm{~h}$ before drug treatment. The cell proliferation activity was detected at the gradient dilution concentrations of PCA $\left(0.1,0.3,1,3,10,30,100,200 \mu \mathrm{mol} \mathrm{L}^{-1}\right)$ in the time course of 24,48 , and $72 \mathrm{~h}$, respectively. After the drug incubation, the cell medium was removed and washed twice with PBS solution, after which $100 \mu \mathrm{L}$ of fresh cell medium containing $20 \mu \mathrm{L}$ MTT solution was added to each well and incubated at $37^{\circ} \mathrm{C}, 5 \% \mathrm{CO}_{2}$ incubator for $4 \mathrm{~h}$. Cell plates were centrifuged at $1000 \mathrm{rpm}, 10 \mathrm{~min}$, in order to make the formazan crystals adhere to the flat plate bottom, then the supernatants were discarded carefully, and $100 \mu \mathrm{L}$ of DMSO was added to each well. Plates were shaken on a plate shaker with the shaking speed of 900 shakes $\mathrm{min}^{-1}$, for 10 min to make sure that the formazan crystals were thoroughly dissolved. The plate absorbance (A) was measured at a wavelength of $570 \mathrm{~nm}$ by a microplate reader. The cell viability was calculated using Equation 1.

$$
\text { Cell viability } \%=100-\left(A_{\text {vehicle }}-A_{\text {drug }}\right) / A_{\text {vehicle }} \times 100
$$

where $A$ is the absorbance value detected at the wavelength of $570 \mathrm{~nm}$. The experiments were performed in triplicates and repeated three times.

\section{Melanin content determination}

HFM cells were pre-treatment with the indicated dosage of PCA $(1,3,10,30$ and 100 $\mu \mathrm{mol} \mathrm{L}-1)$ and vitamin $\mathrm{C}\left(100 \mu \mathrm{mol} \mathrm{L}^{-1}\right)$ respectively for 2 hours, and then $0.15 \mu \mathrm{mol} \mathrm{L}^{-1}$ of alpha-melanocyte-stimulating hormone $(\alpha-\mathrm{MSH})$ was added and the cells were incubated for 24 and $48 \mathrm{~h}$, respectively. Melanin content was determined according to the method of Wojcik et al. (9). HFM cells were counted by the blood cell counter (repeated four times to get the mean value). The cells were washed twice with PBS solution, then $200 \mu \mathrm{L}$ of double steaming water was added to suspend the cells. $1 \mathrm{~mL}$ of the mixed solution of ethanol and ether (1:1) was added, left at room temperature for $15 \mathrm{~min}$, and centrifuged for $5 \mathrm{~min}$ (3000 rpm $\mathrm{min}^{-1}$ ). The supernatant was discarded and $1 \mathrm{~mL}$ of $1 \mathrm{~mol} \mathrm{~L}^{-1} \mathrm{NaOH}$ (containing $10 \%$ DMSO) was added to the tube to dissolve the melanin at $80^{\circ} \mathrm{C}$ for $30 \mathrm{~min}$. Finally, $4 \mathrm{~mL}$ of double steaming water was added to dilute $\mathrm{NaOH}$ to $0.2 \mathrm{~mol} \mathrm{~L}^{-1}$. The absorbance of melanin was determined by spectrophotometer at $475 \mathrm{~nm}$ using Equation 2. Each experiment was repeated four times.

Relative melanin content $\%=\left(A_{475} /\right.$ cell numbers in the related group $) /$

$$
\left(A_{475} / \text { cell numbers in alone } \alpha \text {-MSH induced group }\right) \times 100
$$

\section{Tyrosinase activity determination}

HFM cells were pre-treated with the indicated dosage of PCA $(1,3,10,30$ and $100 \mu \mathrm{mol}$ $\left.\mathrm{L}^{-1}\right)$ and vitamin $\mathrm{C}\left(100 \mu \mathrm{mol} \mathrm{L}{ }^{-1}\right)$ respectively for 2 hours, and then $0.15 \mu \mathrm{M}$ of $\alpha$-MSH was after which the cells were incubated for $24 \mathrm{~h}$. The determination of tyrosinase activity was done according to Kim et al. (10) and with a little improvement of the method. Cells were harvested and counted, washed with PBS twice, after which $1 \mathrm{~mL}$ of $0.5 \%$ sodium deoxycholate lysate was added and the cells suspended and placed at $4{ }^{\circ} \mathrm{C}$ for $1 \mathrm{~h}$, followed by centrifuge at $4{ }^{\circ} \mathrm{C}\left(10000 \mathrm{rpm} \mathrm{min}{ }^{-1}\right)$ for $20 \mathrm{~min}$ to obtain the supernatant containing tyrosinase. $1 \mathrm{~mL}$ of supernatant was added to $3.0 \mathrm{~mL}\left(2.5 \mathrm{~mol} \mathrm{~L}^{-1} \mathrm{~L}\right.$-dopa, $0.1 \mathrm{~mol} \mathrm{~L}^{-1} \mathrm{PBS}, \mathrm{pH}$ 6.8) of L-dopamine reaction solution and incubated at $37^{\circ} \mathrm{C}$ for 20 minutes. The absorbance of dopa pigments was 
B. Li et al:: Exploring the potential effect and mechanisms of protocatechuic acid on human hair follicle melanocytes, Acta Pharm. 70 (2020) 539-549.

determined by spectrophotometer at $475 \mathrm{~nm}$ using Equation 3. At the same time, the automatic oxidation of L-dopa was used for correction. Each experiment was repeated 4 times.

Tyrosinase activity $\%=\left(A_{475} / \mathrm{cell}\right.$ numbers in the related group $) /$

$\left(A_{475} /\right.$ cell numbers in alone $\alpha$-MSH induced group $) \times 100$

\section{Western blot analysis}

HFM cells were seeded in 48 -well plates at a density of $1 \times 10^{5}$ cells per well, cultured at $37^{\circ} \mathrm{C}, 5 \% \mathrm{CO}_{2}$ for $24 \mathrm{~h}$. Following day, the cells were pre-treated with the indicated dosage of PCA $\left(1,10\right.$ and $\left.100 \mu \mathrm{mol} \mathrm{L}^{-1}\right)$ and vitamin $C\left(100 \mu \mathrm{mol} \mathrm{L}^{-1}\right)$ respectively for 2 hours, after which $0.15 \mu \mathrm{M} \alpha$-MSH was added to each well and incubated for $72 \mathrm{~h}$. The total proteins were extracted from the cells using Radio Immunoprecipitation Assay buffer (Sigma Aldrich, USA) and quantified using the BCA kit (Thermo Fisher Scientific, USA). The proteins were separated by $10 \%$ SDS-PAGE gel and transferred to PVDF membrane (Millipore, USA). The membrane was blocked with $5 \%$ non-fat milk for $1 \mathrm{~h}$ at room temperature (r.t.) and incubated overnight with primary antibody (dilution ratio, 1:1000 for MITF and TRP-1; 1:500 for TRP-2; 1:5000 for $\beta$-actin) at $4{ }^{\circ} \mathrm{C}$. The membrane was washed three times with $1 \times$ TBST and incubated with the related secondary antibodies for $1 \mathrm{~h}$ at r.t. The membrane was washed three times with $1 \times$ TBST, then exposed with the Chemiluminescent Substrate (Thermo Fisher Scientific, USA) and imaged on the instrument of BioRad ChemiDoc XRS+ (BioRad, USA).

\section{Quantitative reverse transcription-polymerase chain reaction (RT PCR) analysis}

HFM cells were seeded into 48-well plates at a density of $1 \times 10^{5}$ cells per well, then treated with PCA $\left(1,10\right.$ and $\left.100 \mu \mathrm{mol} \mathrm{L}^{-1}\right)$ and vitamin $\mathrm{C}\left(100 \mu \mathrm{mol} \mathrm{L}^{-1}\right)$ respectively for 2 hours, after which $0.15 \mu \mathrm{M} \alpha$-MSH was added to each well and incubated for $72 \mathrm{~h}$. Total RNA samples were extracted with Trizol reagent (Invitrogen, Thermo Fisher Scientific, USA) following the manufacturer's protocols. Single-stranded cDNA was obtained via reverse transcribe of the total RNA $(1 \mu \mathrm{g})$ with the SuperScript ${ }^{\circledR}$ III First-Strand Synthesis System (18080051, Thermo Fisher Scientific, USA). The cDNA was amplified in an automated thermal cycler (MyGene Series Thermal Cycler MG96G, China). The primer sequences were as follows: MITF (438 bp): forward 5'-CAAATGGCAAATACGTTACCCG-3', reverse 5'-CTCCCTTTTTATGTTGGGAAGGT-3'; TRP1 (499 bp): forward 5'-TACAGTGGAAGGTTACAGTG-3', reverse 5'-AGCATAGCGTTGATAGTGAT-3'; TRP2 (444 bp): forward 5'-ATAACTTGGCTCACTCCTTC-3', reverse 5'-CTTCCTCCGTGTATCTCTTG-3'; $\beta$-actin (446 bp): forward 5'-GAGACCTTCAACACCCCGC-3', reverse 5'-CCACAGGATTCCATACCCAA-3'. The $\beta$-actin gene was amplified separately as an internal control to normalize the gene expression in the samples. The PCR conditions were $5 \mathrm{~min}$ at $94{ }^{\circ} \mathrm{C}$ followed by 32 cycles of 20 s at $94^{\circ} \mathrm{C}, 20 \mathrm{~s}$ at $60^{\circ} \mathrm{C}, 1 \mathrm{~min}$ at $72{ }^{\circ} \mathrm{C}$, and $10 \mathrm{~min}$ at $72{ }^{\circ} \mathrm{C}$. Then PCR products were electrophoresed at $1.5 \%$ agarose gel and analysed with a UV trans-illuminator.

\section{Anti-oxidative marker detection}

SOD and GSH activities were determined by a commercially available kit according to the manufacturer's protocol. SOD reaction was detected at $550 \mathrm{~nm}$ (Thermo Scientific 
Multiskan GO microplate reader, USA) and the values were expressed as $\mathrm{U} \mathrm{mg}^{-1}$ protein, GSH reaction was measured at $420 \mathrm{~nm}$ and the values were expressed as $\mathrm{mg} \mathrm{g}^{-1}$ protein.

\section{Statistical analysis}

The biological values in the study were displayed as means \pm SD. All the statistical comparisons between the groups were performed by one-way ANOVA with Dunnett's t-test in the GraphPad Prism 6.0 software.

\section{RESULTS AND DISCUSSION}

The chemical structure identification by NMR was ${ }^{1} \mathrm{H}$ NMR $\left(\mathrm{CD}_{3} \mathrm{OD}, 400 \mathrm{MHz}\right) \delta(\mathrm{H})$ ( $J$ in Hz): $7.41(1 \mathrm{H}, \mathrm{d}, J=2.4 \mathrm{~Hz}), 6.80(1 \mathrm{H}, \mathrm{d}, J=8.0 \mathrm{~Hz}), 7.44(1 \mathrm{H}, \mathrm{dd}, J=8.0,2.4 \mathrm{~Hz}) .{ }^{13} \mathrm{C} \mathrm{NMR}$

a)

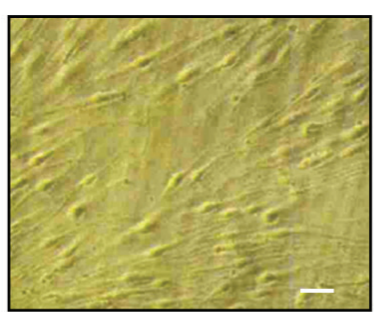

c)

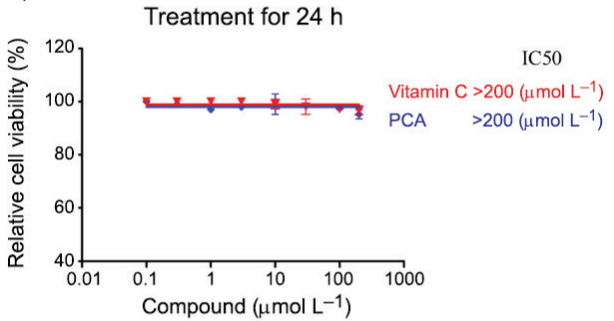

Treatment for $72 \mathrm{~h}$

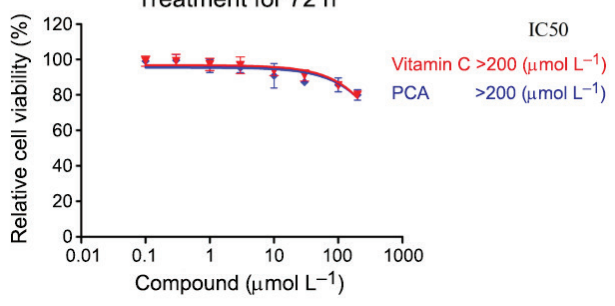

b)
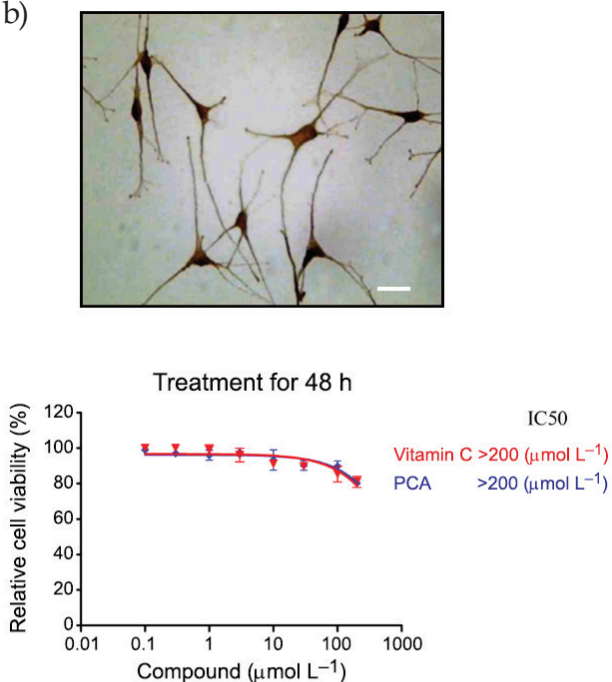

Fig. 2. The effect of PCA on the cell proliferation of HFM: a) the cell morphology of HFM was observed under a photomicroscope (original magnification $\times 200$, scale bar: $100 \mu \mathrm{m}$ ); b) HFM showed a positive signal by dopamine staining (original magnification $\times 200$, scale bar: $100 \mu \mathrm{m}$ ); c) effects of PCA on HFM cell proliferation in the indicated times (24, 48 and $72 \mathrm{~h}$ ). Data are presented using three independent experiments. PCA, protocatechuic acid; HFM, human hair follicle melanocytes. 
$\left(\mathrm{CD}_{3} \mathrm{OD}, 100 \mathrm{MHz}\right) \delta(\mathrm{C}): 123.2,117.8,146.2,151.6,115.9,124.0,170.4$. The purity of PCA analyzed by HPLC showed in Fig. 1. The above data showed that the purchased PCA is correct and has a high purity, which is suitable for its cell biology detection.

To evaluate the effect of PCA on HFM, we have isolated the HFM from normal human head skin, according to the isolation method of Tobin et al. (8). After 1 week, we have imaged the cell morphology and stained the cells with dopamine (Fig. 2). The HFM morphology showed that there are 3 to 5 dendrites in epidermal melanocytes, with smaller cell bodies and stronger refractive index (Fig. 2a), while the dopamine staining showed a positive signal (Fig. 2b).

To examine the effect of PCA on the cell proliferation of HFM, we used 8 gradient dilution concentrations of PCA $\left(0.1,0.3,1,3,10,30,100,200 \mu \mathrm{mol} \mathrm{L} \mathrm{L}^{-1}\right)$ to detect the cell proliferation changes at the time course of 24,48 , and $72 \mathrm{~h}$, respectively. The cells were seeded to 96 -well plate at a density of 5000 cells per well, and the cell viability was measured by MTT assay. From Fig. 2, we can see that PCA treatment did not significantly inhibit the cell proliferation of HFM in a concentration- or time-dependent manner (Fig. 2c), which was similar to the activity of vitamin C. This result suggested that PCA could not induce the cytotoxicity of HFM in vitro culturing at the treatment time of $72 \mathrm{~h}$ (Fig. 2c).

Next, we have detected the effect of PCA on the synthesis of melanin in HFM cels, according to the detection method of Wojcik et al. (9). In the experiment, we have detected the effect of PCA on the synthesis of melanin in five different concentrations $(1,3,10,30$, $100 \mu \mathrm{mol} \mathrm{L}^{-1}$ ) at 24 and $48 \mathrm{~h}$ respectively. As shown in Fig. 3, PCA has significantly reduced the $\alpha$-MSH-induced melanin content in a dose-depended manner compared to the $\alpha$ MSH-induced melanin synthesis group with the $I C_{50}$ of 8.9 and $2.6 \mu \mathrm{mol} \mathrm{L}-1$ at the treatment time of 24 and 48 h respectively $(p<0.05, p<0.01, p<0.001$, Fig. 3). Vitamin $C$ had also significantly decreased the synthesis melanin at 24 and $48 \mathrm{~h}$ treatment at the concentration of $100 \mu \mathrm{mol} \mathrm{L}-1$ (Fig. 3).

Since PCA has a similar parent nucleus structure to many hydroxybenzoate tyrosinase inhibitors, we decided to measure the effect of PCA for the tyrosinase activity on

a)

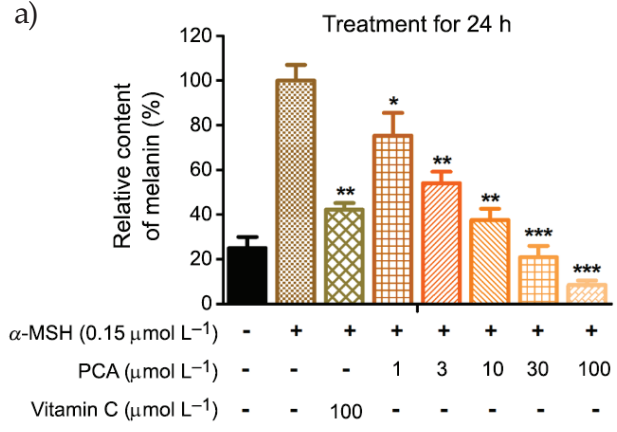

b)

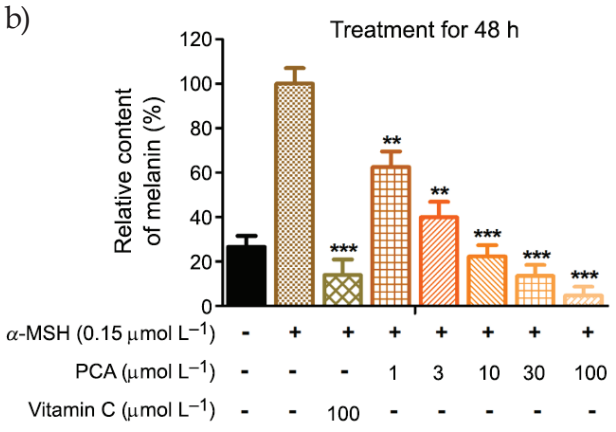

Fig. 3. The effect of PCA on melanin contents: a) relative melanin content after $24 \mathrm{~h}$ treatment of PCA; b) relative melanin content after $48 \mathrm{~h}$ treatment of PCA. Values were calculated relative to alone $\alpha$-MSH induced group and represented the mean \pm SD of three independent replicates. ${ }^{*} p<0.05$, ${ }^{* *} p<0.01,{ }^{* * *} p<0.001$ vs. alone $\alpha$-MSH induced group. PCA, protocatechuic acid. 


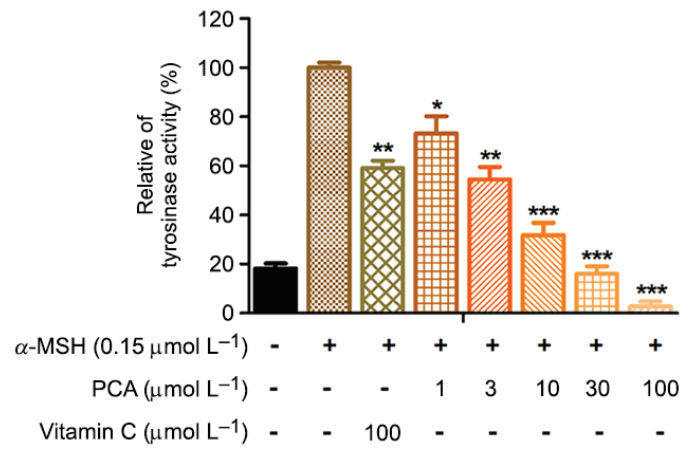

Fig. 4. The effect of PCA on tyrosinase activity. Values were calculated relative to alone $\alpha$-MSH induced group and represented the mean \pm SD of three independent replicates. ${ }^{*} p<0.05,{ }^{* *} p<0.01$, ${ }^{* * *} p<0.001 v$ s. alone $\alpha$-MSH induced group. PCA, protocatechuic acid.

a)

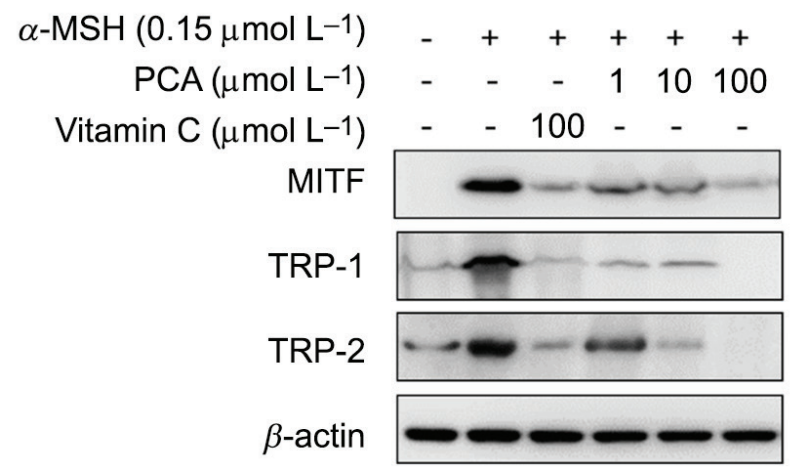

b)

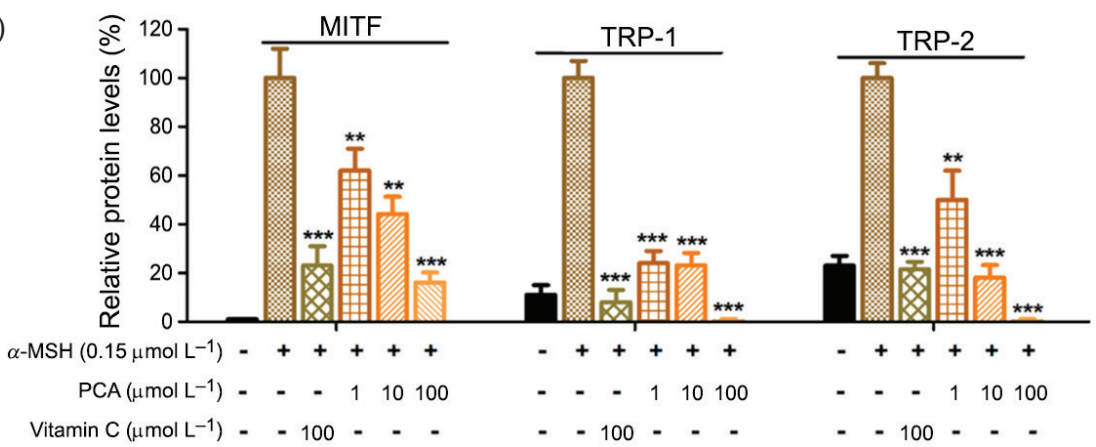

Fig. 5. Effects of PCA on the protein expression of TRP1, TRP2 and MITF: a) the protein expression of TRP1, TRP2 and MITF at the treatment of different PCA concentrations were determined by Western blotting; b) quantification of the protein levels of TRP1, TRP2 and MITF by image J software. $\beta$-actin used as the internal control. Values were calculated relative to alone $\alpha-\mathrm{MSH}$ induced group and represented the mean \pm SD of three independent replicates. Statistical significance is indicated as ${ }^{*} p<0.05,{ }^{* *} p<0.01,{ }^{* * *} p<0.001$ vs. alone $\alpha$-MSH induced group. PCA, protocatechuic acid. 
a)

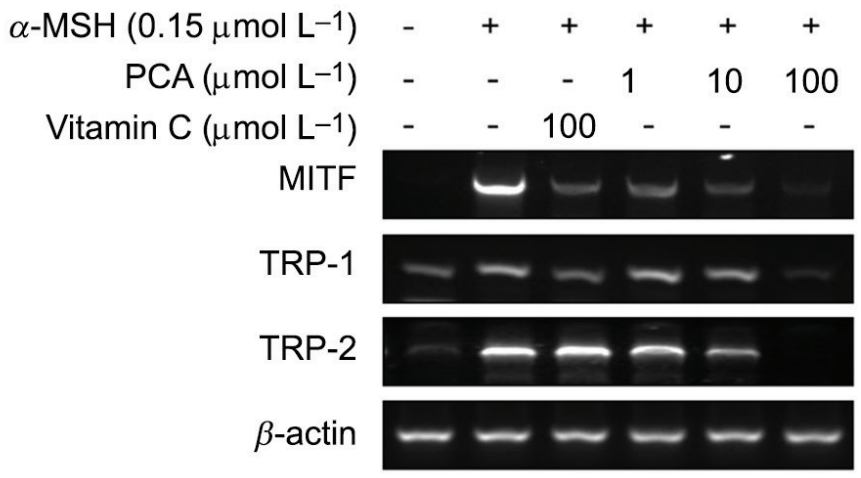

b)

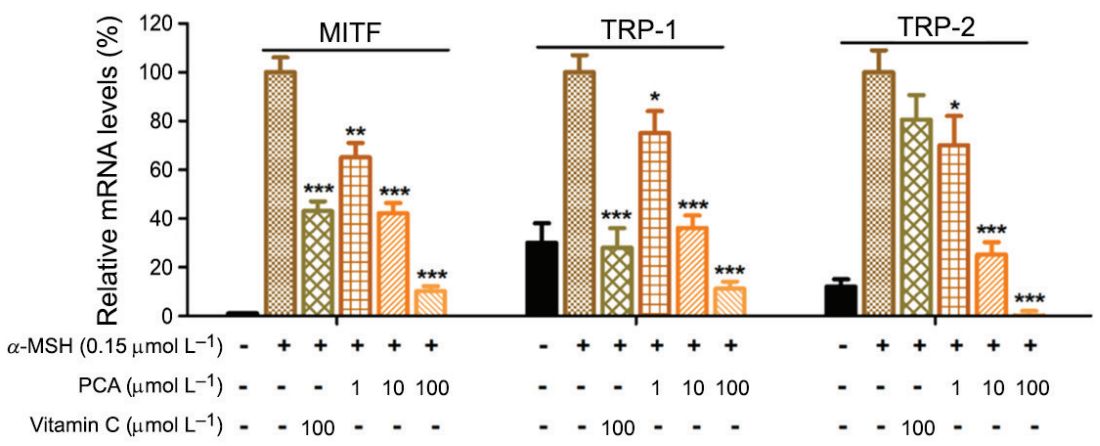

Fig. 6. Effects of PCA on mRNA expression of TRP1, TRP2 and MITF: a) mRNA expression of TRP1, TRP2 and MITF at the treatment of different PCA concentrations were determined by RT-PCR; $b$ ) quantification of the mRNA levels of TRP1, TRP2 and MITF by image J software. $\beta$-actin used as the internal control. Values were calculated relative to alone $\alpha-\mathrm{MSH}$ induced group and represented the mean \pm SD of three independent replicates. Statistical significance is indicated as ${ }^{*} p<0.05,{ }^{* *} p<0.01$, ${ }^{* * *} p<0.001 v s$. alone $\alpha$-MSH induced group. PCA, protocatechuic acid.

HFM (Fig. 4) (10). Compared with $\alpha$-MSH-induced melanin synthesis group, PCA had significantly decreased the activity of tyrosinase in all concentrations of PCA $(1,3,10,30$, $\left.100 \mu \mathrm{mol} \mathrm{L}^{-1}\right)$ in a dose-depended manner $(p<0.05, p<0.01, p<0.001$, Fig. 4$)$, with the $I C_{50}$ of $6.4 \mu \mathrm{mol} \mathrm{L}^{-1}$ for PCA inhibition of tyrosinase activity. While the vitamin $C$ treated group has also shown a reduced activity of tyrosinase at the concentration of $100 \mu \mathrm{mol} \mathrm{L}^{-1}$, comparable to the $\alpha$-MSH-induced melanin synthesis group ( $p<0.01$, Fig. 4).

To further investigate the molecular mechanisms of PCA on melanogenesis regulation, the levels of melanogenic genes and proteins were examined (11-15). As shown in Fig. 5, the effect of PCA on the expression of TRP-1, TRP-2 and MITF proteins was assessed with the Western blot method. The result showed that PCA had significantly inhibited $\alpha-$ MSH-induced expression of TRP-1, TRP-2 and MITF in HFM cells $(p<0.01, p<0.001$, Fig. 5). In addition, we have also detected the mRNA expression of TRP-1, TRP-2 and MITF under the conditions of $\alpha$-MSH triggered by RT-PCR (Fig. 6). From Fig. 6, we can see that PCA treatment had significantly decreased TRP-1, TRP-2 and MITF mRNA expression in dose-depended manners ( $p<0.05, p<0.01, p<0.001$, Fig. 6). Taken above, the result shows 
B. Li et al:: Exploring the potential effect and mechanisms of protocatechuic acid on human hair follicle melanocytes, Acta Pharm. 70 (2020) 539-549.

Table I. Effects of PCA on the levels of SOD and GSH in HFM

\begin{tabular}{cccc}
\hline Group & Dosage $(\mu \mathrm{M})$ & SOD $\left(\mathrm{U} \mathrm{mg}^{-1}\right.$ protein $)$ & GSH $\left(\mathrm{mg} \mathrm{g}^{-1}\right.$ protein $)$ \\
\hline Control & - & $137.70 \pm 7.89$ & $6.36 \pm 0.32$ \\
\hline \multirow{2}{*}{ PCA } & 1 & $140.95 \pm 7.18$ & $6.95 \pm 0.95$ \\
& 10 & $159.50 \pm 11.13^{*}$ & $7.68 \pm 1.23^{*}$ \\
& 30 & $177.95 \pm 9.51^{* *}$ & $8.54 \pm 1.66^{* *}$ \\
& 100 & $199.42 \pm 19.23^{* *}$ & $9.72 \pm 1.43^{* *}$ \\
\hline
\end{tabular}

PCA - protocatechuic acid, HFM - human hair follicle melanocytes.

Data are expressed as mean $\pm \mathrm{SD}, n=3$.

Statistical significance is indicated as ${ }^{*} p<0.05,{ }^{* *} p<0.01$ vs. control group.

that the inhibitory effects of PCA on melanogenesis may be related to the down-regulation of melanogenic genes and proteins (e.g. TRP-1, TRP-2 and MITF).

The two important antioxidant enzyme SOD and GSH are often used to evaluate the ability of anti-oxidative (7). In Table I, the result shows a significant difference in antioxidant levels after PCA treatment for $24 \mathrm{~h}$ in HFM cells. From Table I, we can see that, PCA significantly increased the activity of SOD and GSH in a dose-dependent manner (1-100 $\mu \mathrm{mol} \mathrm{L}-1, p<0.05, p<0.01)$, which implies that the action mechanism of PCA may be related to its anti-oxidative effects.

\section{CONCLUSIONS}

PCA could reduce $\alpha$-MSH-induced melanin synthesis, inhibit the tyrosinase activity, down-regulate of the expression of TRP-1, TRP-2 and MITF, and increase the levels of SOD and GSH in HFM cells, with almost no cytotoxicity to HFM. Thus, the findings implied that PCA can be used as a potential depigmenting agent for the therapy of melanin overexpressed disease or skin whitening.

Acknowledgements. - This study was supported by the Department of Plastic and Aesthetic laser surgery, The people's Hospital of Hunan province, 1st Affiliated Hospital of Hunan Normal University, Hunan, China.

\section{REFERENCES}

1. X. T. Truong, S. H. Park and Y. G. Lee, Protocatechuic acid from pear inhibits melanogenesis in melanoma cells, Int. J. Mol. Sci. 18 (2017) 1-10; https://doi.org/10.3390/ijms18081809

2. K. Krzysztoforska, D. Mirowska-Guzel and E. Widy-Tyszkiewicz, Pharmacological effects of protocatechuic acid and its therapeutic potential in neurodegenerative diseases: review on the basis of in vitro and in vivo studies in rodents and humans, Nutr. Neurosci. 22 (2017) 72-82; https://doi.or g/10.1080/1028415X.2017.1354543

3. R. Hornedo-Ortega, M. A. Álvarez-Fernández, A. B. Cerezo, T. Richard, A. M. Troncoso and M. C. García Parrilla, Protocatechuic acid: inhibition of fibril formation, destabilization of pre-formed 
fibrils of amyloid- $\beta$ and $\alpha$-synuclein, and neuroprotection, J. Agric. Food Chem. 64 (2016) 77227732; https://doi.org/10.1021/acs.jafc.6b03217

4. R. Arslan, S. Aydin and D. Nemutlu Samur, The possible mechanisms of protocatechuic acid-induced central analgesia, Saudi Pharm. J. 26 (2018) 541-545; https://doi.org/10.1016/j.jsps.2018.02.001

5. F. V. Filipp, S. Birlea and M. W. Bosenberg, Frontiers in pigment cell and melanoma research, Pigment Cell Melanoma Res. 31 (2018) 728-735; https://doi.org/10.1111/pcmr.12728

6. P. Janiani, P. R. Bhat and V. A. Trasad, Evaluation of the intensity of gingival melanin pigmentation at different age groups in the Indian population: An observational study, J. Indian. Soc. Pedod. Prev. Dent. 36 (2018) 329-333; https://doi.org/10.4103/ JISPPD.JISPPD_192_17

7. W. C. Liao, Y. T. Huang and L. P. Lu, Antioxidant ability and stability studies of 3-O-ethyl ascorbic acid a cosmetic tyrosinase inhibitor, J. Cosmet. Sci. 69 (2018) 233-243; https://doi.org/10.1102/30311899

8. D. J. Tobin, S. R. Colen and J. C. Bystryn, Isolation and long term culture of human hair follicle melanocytes, J. Invest. Dermatol. 104 (1995) 86-89; https://doi.org/10.1111/1523- 1747.ep12613573

9. S. Wojcik, D. Weidinger and S. Ständer, Functional characterization of the extranasal OR2A4/7 expressed in human melanocytes, Exp. Dermatol. 27 (2018) 1216-1223; https://doi.org/10.1111/exd.13764

10. H. Y. Kim, S. Kishor Sah and S. Choi, Inhibitory effects of extracellular superoxide dismutase on ultraviolet B-induced melanogenesis in murine skin and melanocytes, Life Sci. 210 (2018) 201-208; https://doi.org/10.1016/j.lfs.2018.08.056

11. M. Otreba, J. Rok, E. Buszman and D. Wrzesniok, Regulation of melanogenesis: the role of cAMP and MITF, Postepy Hig. Med. Dosw. 66 (2012) 33-40; https://doi.org/10.1024/22371403

12. J. Y. Lee, Y. R. Cho, J. H. Park, E. K. Ahn, W. Jeong, H. S Shin, M. S. Kim, S. H. Yang and J. S. Oh, Anti-melanogenic and anti-oxidant activities of ethanol extract of Kummerowia striata: Kummerowia striata regulate anti-melanogenic activity through down-regulation of TRP-1, TRP-2 and MITF expression, Toxicol. Rep. 6 (2019) 10-17; https://doi.org/10.1016/ j.toxrep.2018.11.005

13. M. Kanlayavattanakul and N. Lourith, Plants and natural products for the treatment of skin hyperpigmentation-a review, Planta Med. 84 (2018) 988-1006; https://doi.org/10.1055/a-0583-0410

14. M. Otręba, A. Beberok and D. Wrześniok, In vitro melanogenesis inhibition by fluphenazine and prochlorperazine in normal human melanocytes lightly pigmented, DARU J. Pharm. Sci. 26 (2018) 85-89; https://doi.org/10.1007/s40199-018-0206-4

15. A. Tuerxuntayi, Y. Q. Liu, A. Tulake, M. Kabas, A. Eblimit, H. A. Aisa, Kaliziri extract upregulates tyrosinase, TRP-1, TRP-2 and MITF expression in murine B16 melanoma cells, BMC Complement Altern. Med. 14 (2014) 166-170; https://doi.org/10.1186/1472-6882-14-166 